\title{
ARTICLE
}

\section{Some statistics of Ionospheric total electron content variations at mid-latitude zones of Mongolia}

\author{
Baatarkhuu Dagva*, Amarjargal Sharav and Lkhagvajav Chultemiin \\ Department of Astrophysics, Institute of Astronomy \& Geophysics, \\ Mongolian Academy of Sciences, Ulaanbaatar, Mongolia
}

ARTICLE INFO: Received: 15 Feb, 2021; Accepted: 9 Aug, 2021

Abstract: This work is focused on the correlation of ionosphere total electron content (TEC) with solar and geomagnetic activities of the space weather at mid-latitude zone. In our analysis, we investigate the TEC time series obtained from dual-frequency GNSS (Global Navigation Satellite System) observations at three continuous GPS/GNSS stations HOVD (48.00N, 91.66E), CHOB $(48.08 N, 114.53 E)$ and $D A L N(43.56 N, 104.42)$ for 2013. The statistical analyses are performed on 15 minute averaged yearly TEC values, which reveal the semi-annual anomaly and high correlation with the activities of the Sun and the rotation of the Earth. Phase overlapping seasonal variations of TEC and Sunspot, and Solar flux (10.7) indices, and Earth rotations (LOD) and Atmospheric angular moment (AAM) are observed in our data analyses. Sudden ionospheric storm changes in TEC with geomagnetic storm induced by the extreme solar flare and 2013 events were investigated. The result shows that GPS derived TEC behaves as an indicator of these events showing sudden increase in TEC during the event.

Keywords: GPS Ionosphere; total electronic content; ionospheric storm;

\section{INTRODUCTION}

The upper part of the Earth's atmosphere is occupied by ionosphere, which is rich in ions and electrons. Its is at an altitude of $50-1000 \mathrm{~km}$ above the Earth's surface $[1,2]$. An ionospheric storm is a rapidly intensifying stream of highenergy electrons with varying densities produced from the Sun. These are classified as positive or negative storms, with positive storms having high electron densities and negative storms having low electron densities [2]. It is determined by the total electron content
(TEC) and is the main indicator used for recording and comparing the intensity of ionospheric storms. Also, one of the important parameters that determine the state and properties of the ionosphere is the total electron content. The TEC values are mainly influenced by the following factors: solar wind, changes in solar activity, flare in the solar atmosphere, massive solar corona eruptions, high-energy solar particles, extreme ultraviolet solar radiations, and geomagnetic storms $[3,4]$.

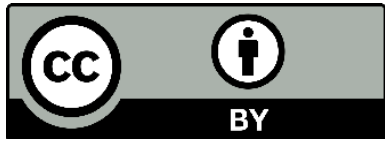

The Author(s). 2021 Open access This article is distributed under the terms of the Creative Commons Attribution 4.0 International License (https://creativecommons.org/licenses/by/4.0/), which permits unrestricted use, distribution, and reproduction in any medium, provided you give appropriate credit to the original author(s) and the source, provide a link to the Creative Commons license, and indicate if changes were made. 
The amount of ionization depends on the geographical location and time, and varies in the equatorial, low-, mid-latitude, and polar regions. Accordingly, ionosphere is divided into three categories: low latitude, medium latitude, and polar region ionosphere.

\section{MATERIALS AND METHODS}

\section{Methodology to derive total electron content from GPS}

We used GPS observation data from HOVD (48.00N, 91.66E), CHOB $(48.08 \mathrm{~N}$, $114.53 \mathrm{E})$ and DALN $(43.56 \mathrm{~N}, 104.42)$ stations of the Astronomical Observatory of the Institute of Astronomy and Geophysics to determine the TEC and its changes. The effects of interactions are studied through the state of the ionosphere [4]. Ionized particles and free electrons in the ionosphere have the greatest effect on the propagation of radio waves. TEC is determined by the total number of electrons in a cylindrical tube with a cross-section of one square meter
The corresponding ionosphere of interest in our country belongs to the middle latitude zone. Advances in the mid-latitude ionosphere research have recently drawn great interest and has been actively investigated by many authors $[4,6]$.

$$
\begin{aligned}
& T E C_{P}=\frac{1}{40.3} \frac{f_{1}^{2} f_{1}^{2}}{f_{2}^{2}-f_{1}^{2}}\left(P_{2}-P_{1}-b_{r}+b_{S}\right) ; \\
& T E C_{\phi}=\frac{1}{40.3} \frac{f_{1}^{2} f_{1}^{2}}{f_{2}^{2}-f_{1}^{2}}\left[\left(\Phi_{1}-\Phi_{2}\right)-\varepsilon_{\Phi 12}-b_{r}+b_{S}-\lambda_{1} N_{1}-\lambda_{2} N_{2}\right] ;
\end{aligned}
$$

Here, $P_{1}$ and $P_{2}$ are the code pseudoranges, 40.3 is a constant of the plasma frequency to the electron density $\left(\mathrm{m}^{3} / \mathrm{s}^{2}\right), b_{s}$ and $b_{r}$ are the estimated satellite and receiver biases, respectively, $\varepsilon_{\phi 12}$ is receiver carrier noise in meters, $N_{1,2}$ is integer ambiguity in cycles, $\Phi_{1,2}$ is the carrier phase measurements at $f_{1}$ and $f_{2}, \lambda_{1}$ and $\lambda_{2}$ are the wavelengths at $f_{1}$ and $f_{2}$, where $f_{l}=1.57542 \mathrm{GHz}$ and $f_{2}=1.22760 \mathrm{GHz}$ are the GPS carrier signal frequencies. The largest source of error in GPS positioning is the ionosphere. However, it is possible to determine the ionospheric delay

\section{RESULTS AND DISCUSSION}

\section{TEC seasonal variations and the factors influencing it}

The peak year of the $24^{\text {th }}$ solar cycle, 2013, was the year of high activity, and static analysis witnessed variations of TEC. perpendicular to the ionosphere [1,5]. Its $10^{16}$ electrons $/ \mathrm{m}^{2}$ is denoted by $1 \mathrm{TECu}$ per unit of TEC $\left(1 \mathrm{TECu}=10^{16}\right.$ electrons $\left./ \mathrm{m}^{2}\right)$. The TEC is determined by both code pseudo-range $(\mathrm{P})$ and phase $(\Phi)$ observations for GPS, and the TEC defined by the pseudo-distance is absolute (Equation 1), while the one calculated from the phase measurement is relative (Equation 2). The pseudo-range derived TEC is absolute but noisy, while phase derived TEC is relative and precise but ambiguous. To get a final VTEC, we performed the following steps: Phase leveling, Bias determination and Projecting slant TEC to vertical TEC (VTEC).

in signal propagation using a binary frequency based on this quality due to ionospheric dispersion in the radio signals. The magnitude of this delay is proportional to the total electron content (TEC) integrated along the signal propagation path $[1,2,6]$.

In this study, we compared data from IERS (International Earth Rotation and Reference Systems Service), SOHO (Solar and Heliospheric Observatory), SIDC (Solar Influences Data Analysis Center), and WDCKyoto (World Data Center-Kyoto) $[9,10,11]$. We performed the calculation using the MatLab programme.

Solar activity is determined by the number of spots on the Sun and its solar flux of $10.7 \mathrm{~cm}$. During this year, there were many powerful flares in the solar atmosphere, some of which resulted in the disruption of plasma 
(ionization gas) from the solar corona or the outer atmosphere (corona mass ejection(CME)), and long-dark lines or powerful magnetic fields (Solar filaments) were frequently observed. Days of the sudden ionospheric storms of this year are also recorded, and we compared them to magnetic storms and solar activity. We tracked the progress of the 2013 TEC changes at each station to identify the days of the strongest ionospheric storms. For example, TEC at the DALN GPS station on Figure 1 shows the ionospheric storms and solar events, and geomagnetic storms (its index Dst) that occurred in 2013. Figure 2 shows the color variation of the day-night variation of the annual TEC change at the DALN station.

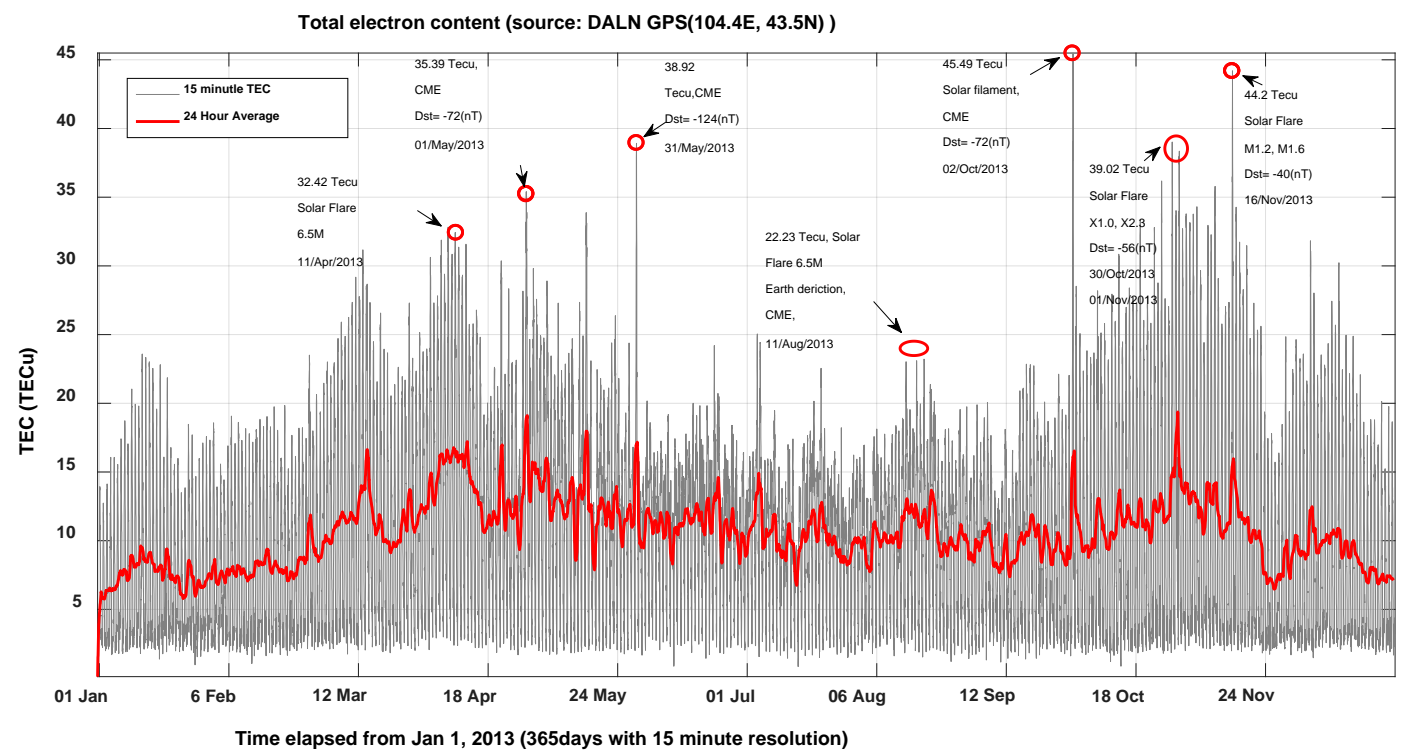

Figure 1. TEC variations at the DALN station for the 2013, overlaid by Ionospheric storm, amplitude of TEC, Geomagnetic storm and Solar flare, CME, Solar filament

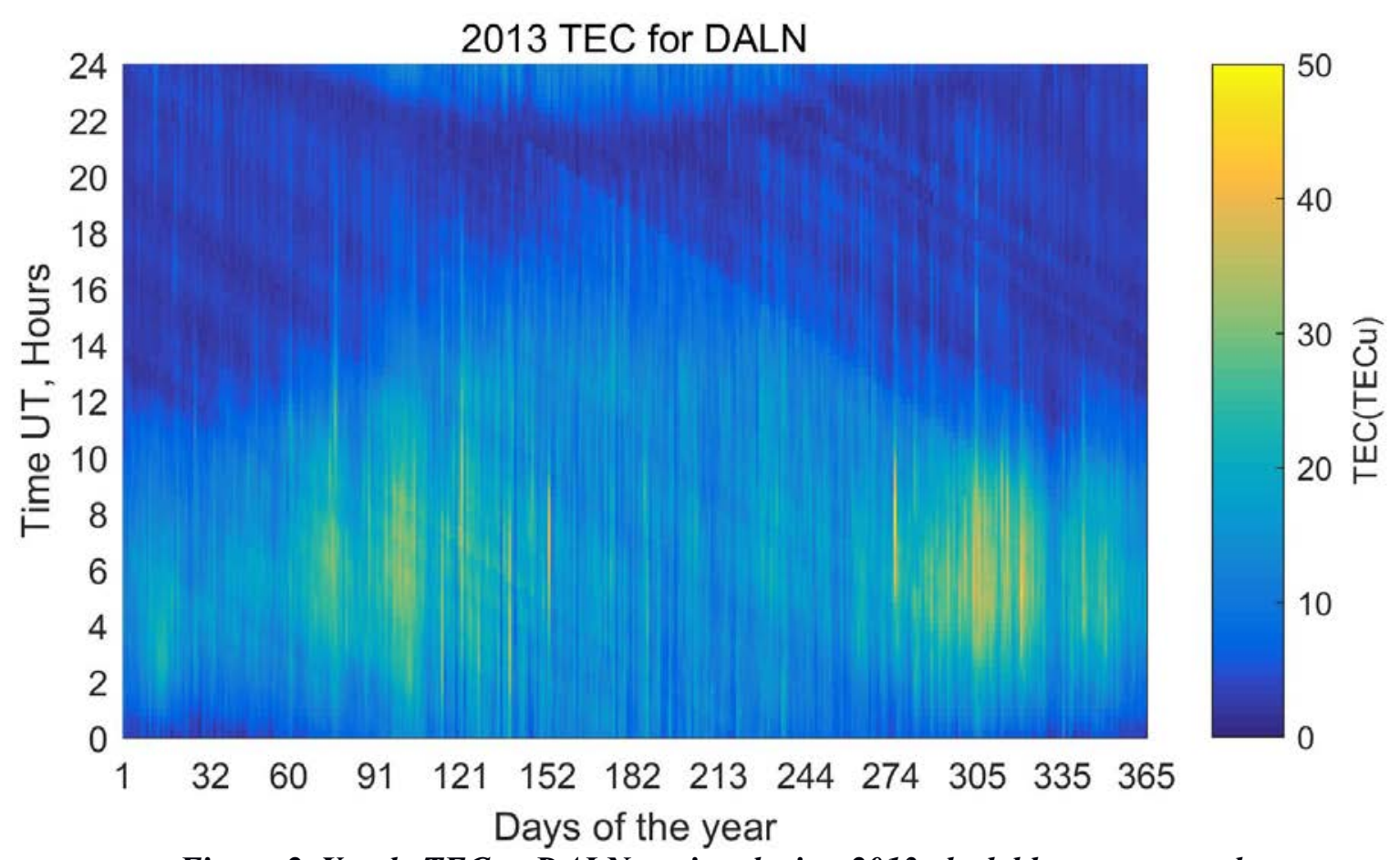

Figure 2. Yearly TEC at DALN station during 2013, dark blue corresponds to night-time TEC and white blue-daytime 


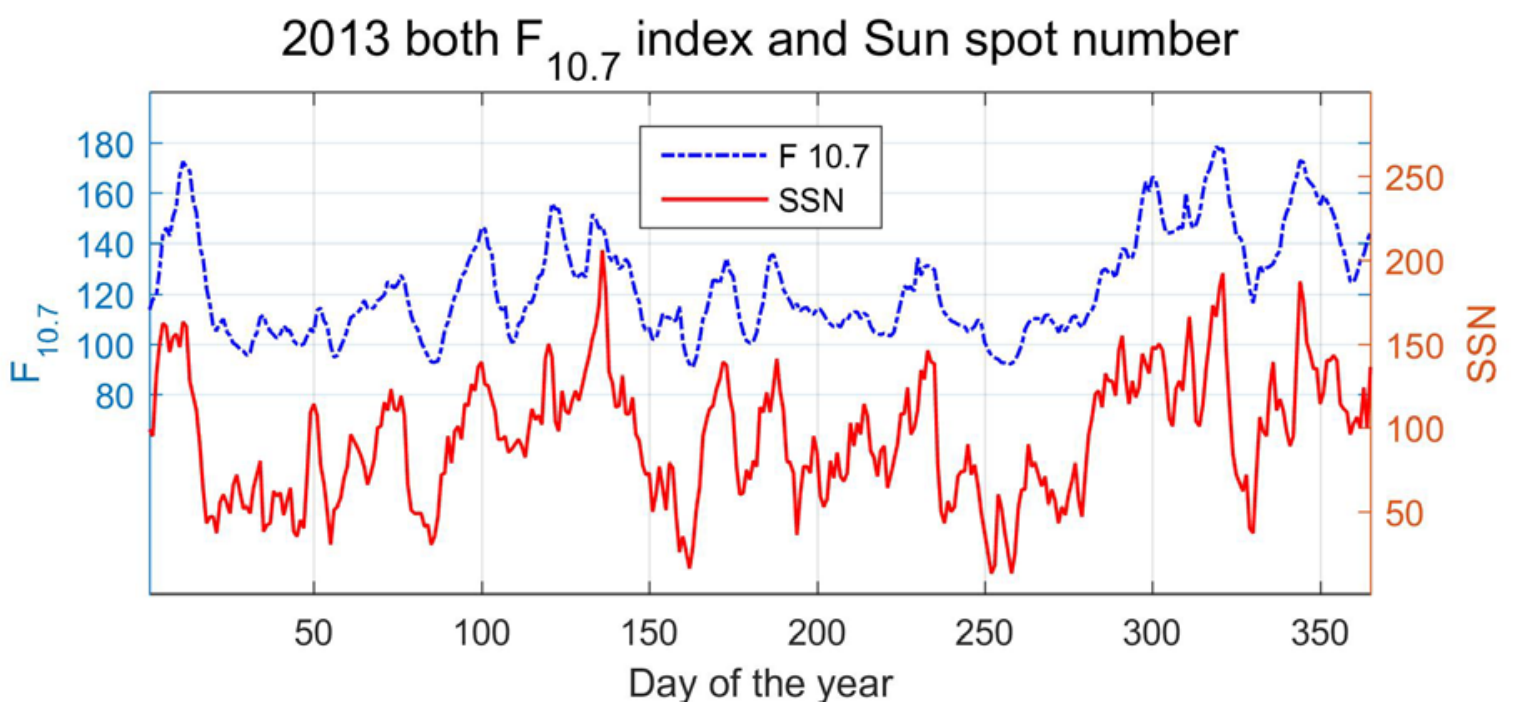

Figure 3. Annual distribution of solar index F10.7 and Sun spot number

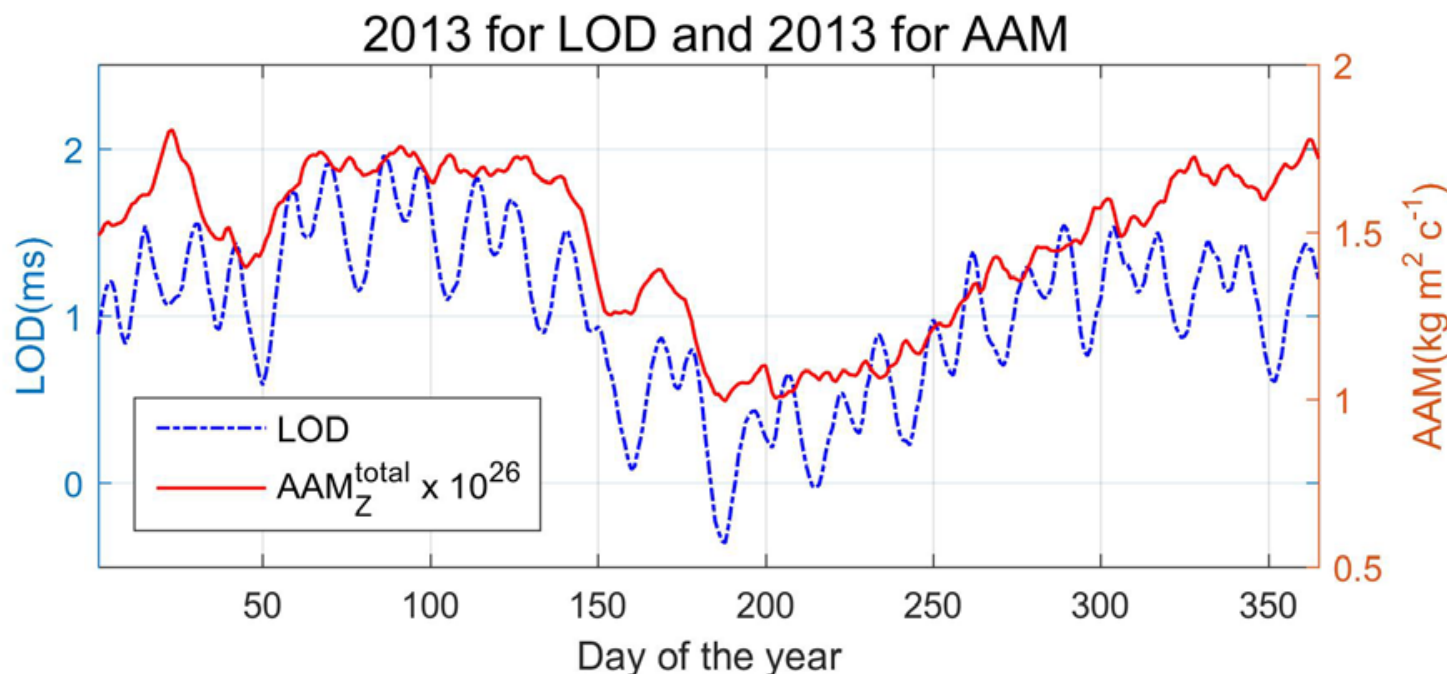

Figure 4. Annual distribution of Earth rotations and Atmospheric angular momentum

Figure 3 shows that the Solar Activity Index, the number of spots, and the $10.7 \mathrm{~cm}$ wavelength of radiation during a given year correspond to the phase intensity of the TEC. This activity increased in March, April and October, November.

We have been working for many years on studies and analyses of the Earth's rotation. It is one of the most important sciences about the Earth, which is related to time, and other many dimensions, and subjects [1]. One of the most important parameters that determine the rotation of the earth is the length of the day. It is internationally abbreviated as "length of day" or LOD (Figure 5). It also has many periodic changes, and is always associated with atmospheric angular momentum (AAM) or the number of rotations $[1,2,13]$.

During the year of interest, the Earth's rotation and atmospheric angular momentum slow down in March and April, accelerate in June and July, and once again slow down again in October and November (Figure 4). This is in phase with the annual changes in the TEC. The annual rate of TEC is always increasing in March and April, decreasing in June and July, and increasing again in October and November.

\section{Monthly variations in TEC}

We have obtained the monthly changes of the TEC in the days of the 2013 ionospheric storm. Figure 5 below shows change in the 
monthly average of October 2013 as observed at each GPS station. The October 2 ionospheric storm was a very special and a powerful storm. The main source of the storm is connected to a thin continuous filament (magnetic filament) in the Sun's photosphere, i.e., a flame that appears as a fine black elongated wire on the Sun's surface [9]. Thus, due to the instantaneous connection of the opposite lines of the magnetic field of the giant flame, the plasma substance is strongly scattered and the corona mass is directed towards the Earth [14].
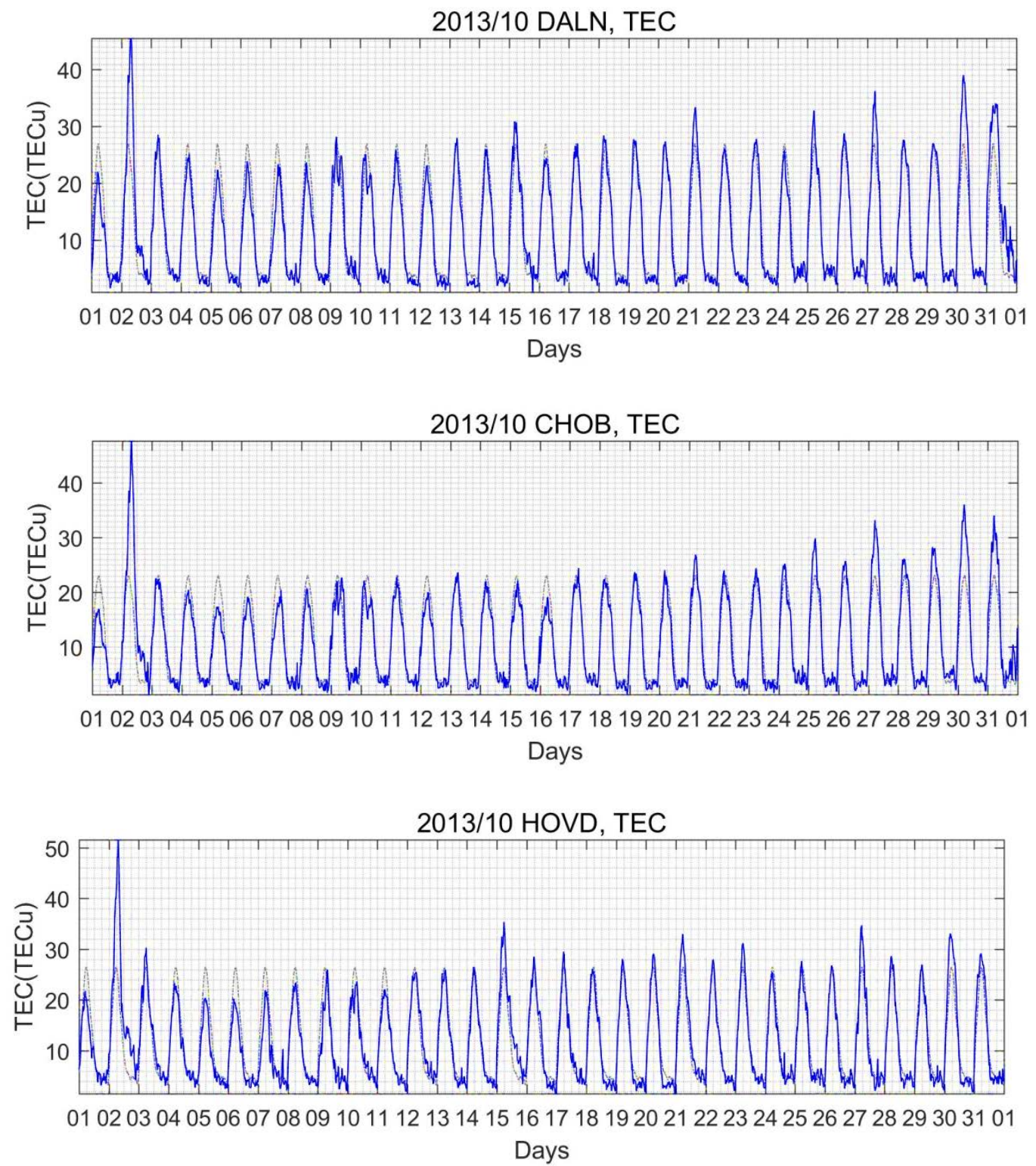

Figure 5. TEC variations in October 2013 at each station. The highest TEC corresponds to the ionospheric storm day on October 2. Dashed line represents the monthly average TEC

Figure 5 shows the TEC time series at the DALN, CHOB, and HOVD GPS stations in October 2013. The intensity of the TEC reached 45.49TECu for DALN, 47.68TECu for CHOB, and 51.53TECu for HOVD, respectively, on October 2, the day of the ionospheric storm. The highest TEC value is observed at the
HOVD station, probably depending on the geomagnetic latitude.

For example, for the HOVD station, where the monthly average TEC was 26.41TECu, TEC reached 51.53TECu on that day, increasing by a factor of 2 . This can be seen in Figure 5 and Table 1 in detail. 
We calculated the mean and maximum TEC values in Table 1 and their deviations at the ionospheric storm days for each station. As seen from Table 1, the value of TEC increased slightly above the monthly average for each station on the days of ionospheric storms, as well as the magnitude of the magnetic storms that occurred during the day and the influence of the Sun $[10,11]$.
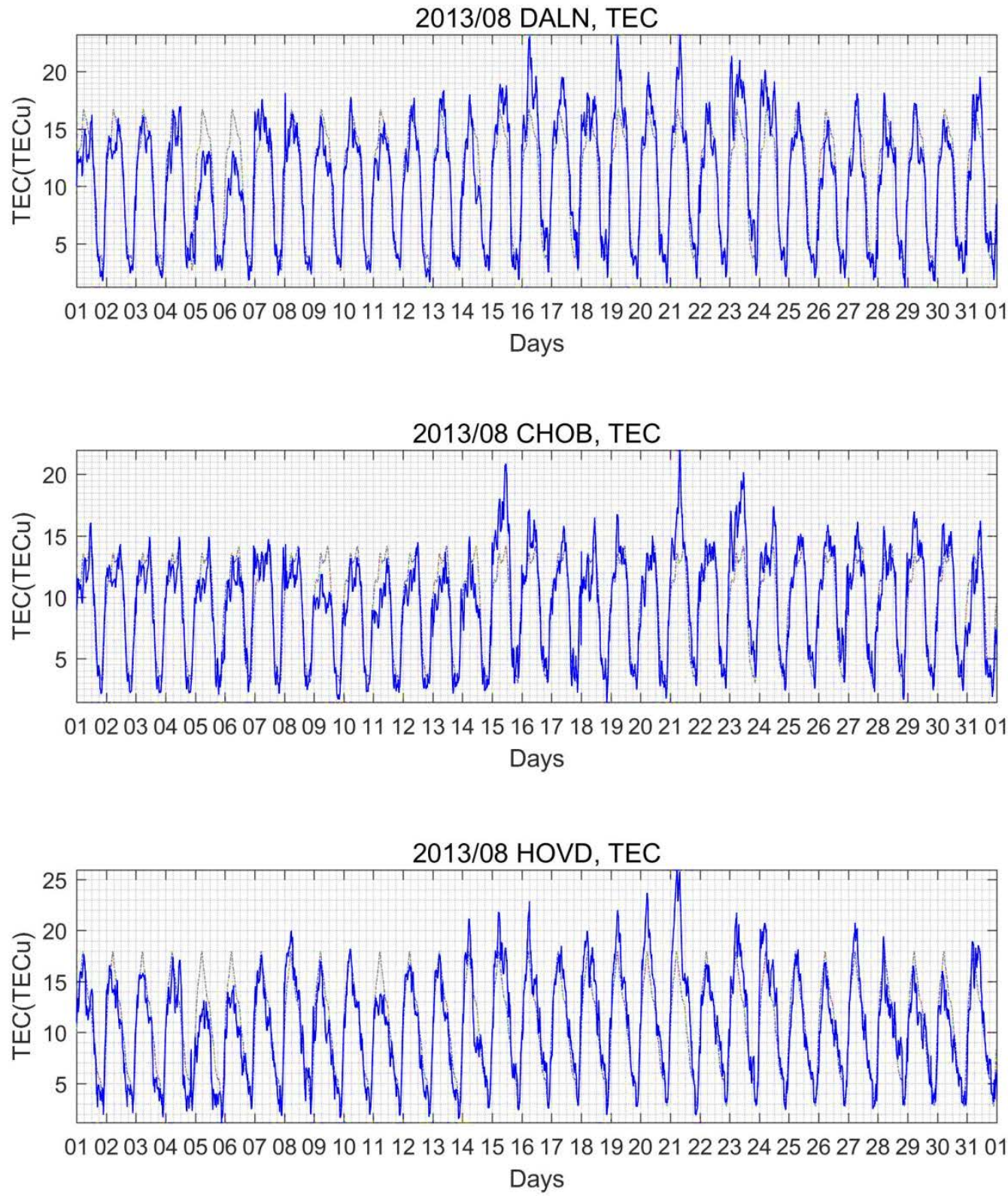

Figure 6. TEC variations at each station in August 2013. The highest TEC corresponds to the ionospheric storm day on August 21. Dashed line represents the monthly average

Figure 6 shows TEC time series at the DALN, CHOB, and HOVD GPS stations in August 2013. The intensity of the TEC reached 23.22TECu for DALN, 21.97TECu for CHOB, and 25.95TECu for HOVD on August 21, the day of the ionospheric storm. The deviation of the TEC from the three station averages varied by about 7-8TECu during the earth-directed CME on August 21, 2013 [11]. 
Table 1. Statistical analysis of TEC of each GPS stations in the days of ionospheric storms during the year 2013

\begin{tabular}{|c|c|c|c|c|c|c|c|c|c|c|c|}
\hline \multirow{2}{*}{$\begin{array}{l}\text { Ionospheric } \\
\text { storm event } \\
\text { in } 2013\end{array}$} & \multicolumn{3}{|c|}{$\begin{array}{c}\operatorname{DALN}(43.56 N, 104.42) \\
{[\mathrm{TECu}]}\end{array}$} & \multicolumn{3}{|c|}{$\begin{array}{c}\mathrm{CHOB}(48.08 N, 114.53 E) \\
{[\mathrm{TECu}]}\end{array}$} & \multicolumn{3}{|c|}{$\begin{array}{c}\operatorname{HOVD}(48.00 N, 91.66 \mathrm{E}) \\
{[\mathrm{TECu}]}\end{array}$} & \multirow{2}{*}{$\begin{array}{c}\text { Dst } \\
(\min , \\
\max ) \\
\text { WDC- } \\
\text { Kyoto }\end{array}$} & \multirow{2}{*}{$\begin{array}{l}\text { Solar events } \\
\text { (SOHO) }\end{array}$} \\
\hline & $\begin{array}{l}\text { TEC } \\
\text { mean }\end{array}$ & $\begin{array}{l}\mathrm{TEC} \\
\max \end{array}$ & $\triangle T E C$ & $\begin{array}{l}\text { TEC } \\
\text { mean }\end{array}$ & $\begin{array}{l}\text { TEC } \\
\max \end{array}$ & $\triangle T E C$ & $\begin{array}{l}\text { TEC } \\
\text { mean }\end{array}$ & $\begin{array}{l}\text { TEC } \\
\max \end{array}$ & $\triangle T E C$ & & \\
\hline 01 May & 21.35 & 35.39 & 14.04 & 15.8 & 25.32 & 9.52 & 28.7 & 41.91 & 13.21 & $-72(\mathrm{nT})$ & CME \\
\hline 01 June & 16.68 & 38.92 & 22.64 & 12.39 & 34.04 & 21.65 & 19.15 & 41.84 & 22.69 & $124(\mathrm{nT})$ & CME \\
\hline $21 \mathrm{Aug}$ & 16.7 & 23.22 & 6.52 & 14.15 & 21.97 & 7.82 & 17.96 & 25.95 & 7.99 & $+28(\mathrm{nT})$ & CME \\
\hline 02 Oct & 26.93 & 45.49 & 18.56 & 23.10 & 47.68 & 24.58 & 26.41 & 51.53 & 25.12 & $-72(\mathrm{nT})$ & $\begin{array}{l}\text { Solar } \\
\text { filaments }\end{array}$ \\
\hline $30 \mathrm{Oct}$ & 26.93 & 39.02 & 12.09 & 23.10 & 36.06 & 12.96 & 26.41 & 33.04 & 6.63 & $-56(\mathrm{nT})$ & $\mathrm{X} 1.0, \mathrm{X} 2.3$ \\
\hline $01 \mathrm{Nov}$ & 27.2 & 38.35 & 11.15 & 25.23 & 29.52 & 4.29 & 27.2 & 37.23 & 10.03 & $-32(\mathrm{nT})$ & CME \\
\hline $16 \mathrm{Nov}$ & 27.2 & 44.2 & 17.0 & 25.23 & 43.71 & 18.48 & 27.2 & 40.98 & 13.78 & $-40(\mathrm{nT})$ & M1.2, M1.6 \\
\hline
\end{tabular}

Depending on the location of the stations, Table 1 shows the difference of mean monthly TEC value between stations HOVD and CHOB which are located apart by $24^{\circ}$ longitude, which

\section{CONCLUSIONS}

All the active dynamic phenomena coming from the Sun to the Earth are interrelated. We made the following conclusions from a study of the monthly, annual, and ionospheric storm abrupt changes in the TEC identified from GPS observation data:

- The total concentration of electrons in the ionosphere, solar activity, and seasonal changes in the Earth's rotation are correlated.

- Ionospheric storms occur on days when the Sun active phenomenon is exposed to geomagnetic storms. Ionospheric storms are an important indicator of the Sun's impact.

- Among three stations, the average monthly values of TEC for HOVD and DALN stations is about 3-4TECu, higher than those determined by $\mathrm{CHOB}$ station, which can be explained by their geomagnetic latitudes. is greater than 3-4TECu, while for HOVD and DALN stations this difference is almost negligible. This can be attributed again to the higher geomagnetic latitudes of HOVD station and lower for the DALN station.

We have presented the main content of this article, some new ideas and innovative results at the Russian-Mongolian international and local scientific conferences.

Acknowledgements. This sudy was funded by the Mongolian Foundation for Science and Technology (Project No. 2018/30). We acknowledge the use of the data provided by IERS (International Earth Rotation and Reference Systems Service), SOHO (Solar and Heliospheric Observatory), SIDC (Solar Influences Data Analysis Center), and WDCKyoto (World Data Center-Kyoto). Authors thank B.Tuguldur, $\mathrm{PhD}$, teacher at the Department of Physics of the National University of Mongolia and other reviewers for their assistance in evaluating this paper. 


\section{REFERENCES}

1. Baatarkhuu D., Amarjargal Sh, (2020), Some results of space weather studies using GPS ionosphere data, Journal of Geophysics and Astronomy, ISSN2709-1546, volume7, pp. 80-86, (in Mongolian).

2. Baasanjav. D, Lkhagvasuren. D,..,(2016), Astronomy, book, ISBN:978-99973-912-6-1, (in Mongolian).

3. Sh .Amarjargal,..., (2016), Space geodetic activities at the Astronomical Observatory of Mongolia. DOI 10.1515/jogs-2016-0009.

4. Cander, Ljiljana R, Ionospheric Space Weather. Springer.ISBN 978-3-31999331-7.

5. Calabia, A., \& Jin, S. (2020), New modes and mechanisms of long -term ionospheric TEC variations from global ionosphere maps. Journal of Geophysical Research: Space Physics, 125, e2019JA027703. https://doi.org/ 10.1029/2019JA027703.

6. He, M., L. Liu, W. Wan, and B. Zhao (2011), A study on the nighttime midlatitude ionospheric trough, J. Geophys. Res., 116, A05315, doi:10.1029/2010JA016252.

7. Patel, N. C., Karia, S. P. and Pathak, K. N. (2017), GPS-TEC Variation during Low to High Solar Activity Period (2010-2014) under the Northern Crest of Indian Equatorial Ionization Anomaly Region. Positioning, 8, 1335.

https://doi.org/10.4236/pos.2017.8200 2.

8. J. U. Kozyra W. B. Manchester,..,(2013), Earth's collision with a solar filament on 21 January 2005: Overview, Journal of geophysical research, space physics, vol. 118, pp.5967-5978, doi:10.1002/jgra.50567.

9. Hintsa Gebreselasse and Gebregiorgis Abraha.(2017), Global Variations of Ionospheric Total Electron Content (TEC) Derived from GPS Global Ionospheric Maps. ISSN: 2220-184X, Volume 9(2):141-161.

10. http://sidc.oma.be/silso/datafiles.

11. https://www.space.com/20621-solarmaximum-sun-storm-photos2013.html.

12. http://wdc.kugi.kyoto-u.ac.jp/dstdir/

13. https://www.iers.org/IERS/EN/Home/ home_node.html

14. Ren, D., \& Lei, J. (2017). A simulation study of the equatorial ionospheric response to the October 2013 geomagnetic storm. Journal of Geophysical Research: Space Physics, 122,9696-9704. https://doi.org/10.1002/ 